\title{
Efecto de la Dosis de Glifosato sobre la Biomasa de Malezas DE BARBECHO AL ESTADO VEGETATIVO Y REPRODUCTIVO ${ }^{1}$
}

\author{
Glyphosate Dose Effect on Weed Biomass at the Vegetative and Reproductive Stage
}

\author{
PURICELLI, E. ${ }^{2}$ y FACCINI, D. ${ }^{3}$
}

\begin{abstract}
RESUMEN - Los experimentos se condujeron en el campo experimental de la Facultad de Ciencias Agrarias ubicado en Zavalla (Argentina) durante 2005 y 2006. E1 objetivo de este trabajo fue estudiar la eficacia de glifosato aplicado al estado vegetativo y reproductivo de Convolvulus arvensis, Oenothera indecora, Iresine diffusa, Parietaria debilis, Rumexparaguayensis y Trifolium repens. El diseño del experimento fue completamente al azar con un arreglo factorial: año, especies, estado reproductivo y vegetativo y dosis de glifosato $48 \%$ (4X, 2X, $1 \mathrm{X}, 1 / 2 \mathrm{X}, 1 / 4 \mathrm{X}, \mathrm{OX}$ ) siendo $\mathrm{X}$ la dosis recomendada $1200 \mathrm{~g}$ i.a. ha ${ }^{-1}$. Se estableció la relación entre la dosis de glifosato y el control de la biomasa de las malezas a través de curvas de dosis respuesta con un modelo log-logístico. Se comparó el grado de tolerancia por medio de la $\mathrm{DL}_{50}$. En ambos estados de las malezas, la mayor $\mathrm{DL}_{50}$ obtenida para I. diffusa indica que de las especies estudiadas ésta es la más tolerante a glifosato. E1 número de especies tolerantes al glifosato es menor al estado vegetativo que al reproductivo.
\end{abstract}

Palabras-clave: dosis-respuesta, glifosato, malezas.

ABSTRACT - Experiments were conducted at the University of Rosario Experimental Farm, Zavalla in 2005 and 2006 to study the effect of glyphosate on the control of Convolvulus arvensis, Oenothera indecora, Iresine diffusa, Parietaria debilis, Rumex paraguayensis and Trifolium repens at the vegetative and reproductive stage. The experiments were established in a complete randomized design with the following factorial arrangement of treatments: year, species, vegetative and reproductive growth stages and glyphosate 48\% (4X,2X,1X,1/2X, 1/4X, OX) being $1 X$ the recommended dose $\left(1,200 \mathrm{~g}\right.$ a.i. ha $\left.\mathrm{m}^{-1}\right)$. The relationship between glyphosate dose and weed biomass control was established with a log-logistic model. The degree of tolerance was compared by $L D_{50}$. In both stages, the higher $L D_{50}$ was obtained for I. diffusa indicating that this is the species most tolerant to glyphosate among those studied. The number of species tolerant to glyphosate is lower at the vegetative stage than at the reproductive stage

Keywords: dose-response, glyphosate, weeds.

\section{INTRODUCCIÓN}

El barbecho es una práctica común en los agroecosistemas y generalmente es necesario realizar un control químico para controlar las malezas en estos sistemas (Derksen et al., 1995). En la región sojera central de Argentina, el barbecho entre cultivos de verano es una práctica usual. La composición y abundancia de las especies de malezas en la comunidad presente en los barbechos de Zavalla (Argentina) fue determinada durante varios años (Puricelli \& Tuesca, 2006). Convolvulus arvensis, Oenothera indecora, Iresine diffusa, Parietaria debilis, Rumex paraguayensis y Trifolium repens son especies presentes en barbechos y son dificiles de controlar según un estudio previo (Faccini \& Puricelli, 2007).

1 Recebido para publicação em 8.7.2008 e na forma revisada em 5.6.2009.

2 Correspondencia: E Puricelli, Cátedra de Terapéutica Vegetal, Facultad de Ciencias Agrarias, Universidad Nacional de Rosario. CC 14 (S 2125 ZAA), Zavalla, Santa Fe, Argentina; Tel: 0341-4970080;<epuri@express.com.ar>. ${ }^{3}$ Cátedra de Malezas, Facultad de CiEncias Agrarias, Universidad Nacional de Rosario. CC 14 (S 2125 ZAA), Zavalla, Santa Fe, Argentina.

Planta Daninha, Viçosa-MG, v. 27, n. 2, p. 303-307, 2009 
Asimismo, algunas de estas especies como $P$. debilisy $T$. repens pueden ser problemáticas al momento de la siembra de los cultivos de verano. Iresine diffusa puede encontrarse también durante todo el ciclo de los cultivos de verano (Papa, 2003).

La tolerancia a un herbicida es la capacidad inherente de una especie para sobrevivir después de un tratamiento herbicida en aplicaciones realizadas con la dosis normal de uso para la mayoría de la especies.

Generalmente, en las especies tolerantes se recomiendan altas dosis de herbicidas y puede en algunos casos haber una sobreestimación de la cantidad requerida para obtener un control adecuado. Dentro de las medidas promisorias para minimizar el consumo de herbicidas se incluye el uso de dosis reducidas (Zoschke, 1994). Sin embargo, si las plantas sobrevivientes son capaces de producir semilla, las poblaciones pueden incrementarse en los años siguientes. Consecuentemente, es importante estudiar en las especies tolerantes el efecto de un amplio rango de dosis de glifosato para determinar su tolerancia relativa al herbicida.

Por otro lado, a medida que las malezas incrementan su tamaño, se tornan menos susceptibles a los herbicidas (Devlin et al., 1991; Klingaman et al., 1991; Blackshaw \& Harker, 1997). En particular glifosato el tamaño de la maleza puede afectar la eficacia (Vanlieshout \& Loux, 2000). A pesar de que el efecto de la especie y estado de desarrollo de la planta es conocido, la dosis de glifosato que permite el control no se conoce para las especies estudiadas en este trabajo.

El objetivo de este trabajo fue estudiar la eficacia de glifosato aplicado al estado vegetativo y reproductivo de $C$. arvensis, $O$. indecora, I. diffusa, $P$. debilis, $R$. paraguayensis y T. repens.

\section{MATERIALES Y MÉTODOS}

Los experimentos se realizaron en 2005 y 2006 en el Campo Experimental de la Facultad de Ciencias Agrarias de la Universidad

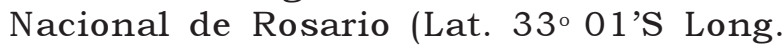
$60^{\circ} 53^{\prime} \mathrm{W}$ ) en macetas ubicadas al aire libre durante la estación de crecimiento de las malezas. El suelo fue obtenido en el sitio de estudio, tamizado a través de un tamiz de $5 \mathrm{~mm}$ y pasteurizado a $80^{\circ} \mathrm{C}$ durante $24 \mathrm{~h}$. Las macetas tenían $18 \mathrm{~cm}$ de diámetro y $8 \mathrm{~kg}$ para especies de menor tamaño, $28 \mathrm{~cm}$ de diámetro y $15 \mathrm{~kg}$ para especies de mayor tamaño y agujeros de drenaje. Las semillas de las malezas fueron recolectadas durante el año anterior a cada experimento y se sembraron en las macetas en mayo (primera siembra) y julio (segunda siembra), de cada año. Las macetas se regaban superficialmente cuando era necesario. Cuando las plántulas poseian aproximadamente 2 a 3 hojas se ralearon a 1 por maceta. El experimento se estableció con un diseño factorial de bloques completos aleatorizados con los siguientes factores: año con 2 niveles, especies con 6 niveles, estado de desarrollo con 2 niveles y dosis de glifosato con 6 niveles (4X, 2X, 1X, 1/ 2X, 1/4X, OX) siendo X la dosis recomendada: 1200 g i.a. ha ${ }^{-1}$ (CASAFE, 2007). Para cada estado se utilizaron 4 repeticiones consistentes en una maceta aleatoriamente asignada a cada tratamiento. Las aplicaciones de glifosato se realizaron a fines de septiembre de cada año con una mochila de presión constante con abanico plano 8003 con una tasa de aplicación de $120 \mathrm{~L} \mathrm{ha}^{-1} \mathrm{y}$ una presión de $270 \mathrm{kPa}$. Cuarenta días después de la aplicación las plantas sobrevivientes se cosecharon y se secaron a $80^{\circ} \mathrm{C}$. El control se determinó a través del peso de cada especie en cada tratamiento en relación al testigo sin aplicación.

Se utilizaron modelos log-logísticos (Seefeldt et al., 1995) para relacionar la dosis de glifosato con la biomasa en por ciento con respecto al testigo sin aplicación. Se evaluaron los coeficientes de determinación, los cuadrados medios, la suma de cuadrados y los residuales. El parámetro $I_{50}$ y la dosis necesaria para controlar el $90 \%$ de la biomasa de las especies de malezas fueron comparados mediante ANOVA y las medias se separaron usando una Prueba de LSD $(P=0,05)$.

\section{RESULTADOS Y DISCUSIÓN}

La habilidad del glifosato para controlar un amplio rango de especies de malezas ha sido descripta en una revisión (Schütte et al., 2004) y en un trabajo previo en la zona del 
presente estudio (Faccini \& Puricelli, 2007). Sin embargo, las especies incluidas en el presente trabajo muestran dificultades de control y se consideran tolerantes. Estas especies mostraron control inadecuado en otros estudios: C. arvensis (Westwood \& Weller, 1997; Chaves et al., 2001), O. indecora (Papa, 2003; Puricelli et al., 2005), R. crispus (Faccini et al., 2005), and T. repens (Puricelli et al., 2004). En particular, P. debilis es una especie tolerante (Puricelli \& Papa, 2006) que se encuentra en muy altas densidades en los barbechos de la región (Puricelli \& Tuesca, 2006).

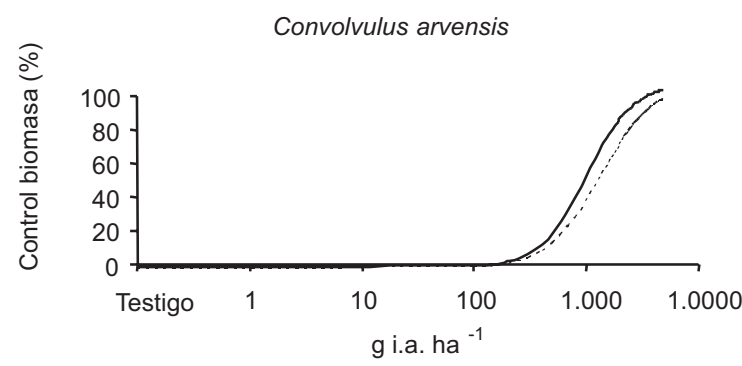

Estado vegetativo: $108+(-1,9-108) /\left(1+(\text { dosis } / 1012)^{\wedge} 2,1 \quad r^{2}=0,97\right.$ Estado reproductivo: $108+(-2,9-108) /\left(1+(\text { dosis } / 1364)^{\wedge} 1,8 r^{2}=0,96\right.$

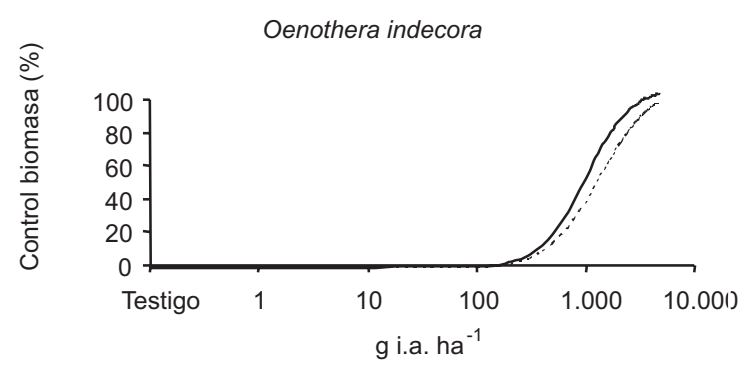

Estado vegetativo: $96,8+(-1,1-96,8) /\left(1+(\text { dosis } / 526)^{\wedge} 1,9 \quad r^{2}=0,98\right.$ Estado reproductivo: $99,5+(-3,9-99,5) /\left(1+(\right.$ dosis $/ 660) \wedge 2,7 r^{2}=0,98$

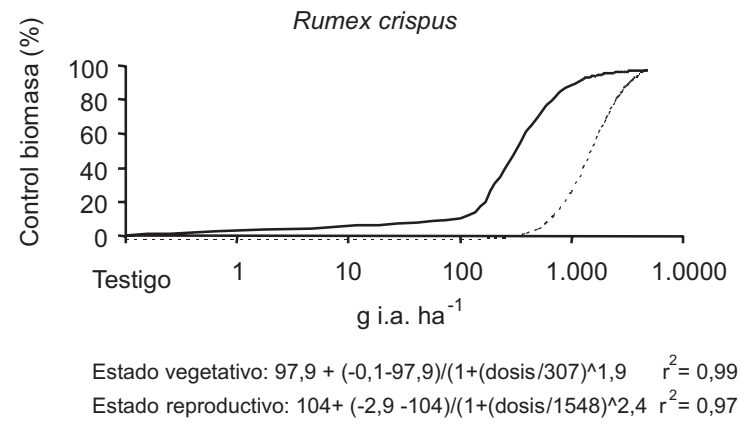

El control de las especies estudiadas no difirió en los dos años del experimento por lo cual los datos se analizaron conjuntamente para los dos años. Los datos se ajustaron en todos los casos al modelo logístico. Las curvas del estado vegetativo se ubicaron siempre por debajo de las del estado reproductivo (Figura 1).

$I_{50}$ es normalmente el mejor estimador posible de la sensibilidad de una planta a un herbicida y es útil para realizar comparaciones entre diferentes experimentos de dosis respuesta (Streibig et al., 1993). Al analizar a través de ANOVA los $\mathrm{I}_{50}$ se detectó una

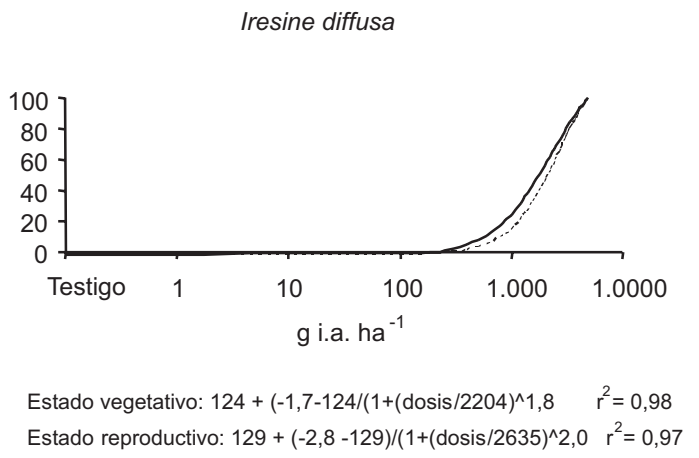

Parietaria debilis

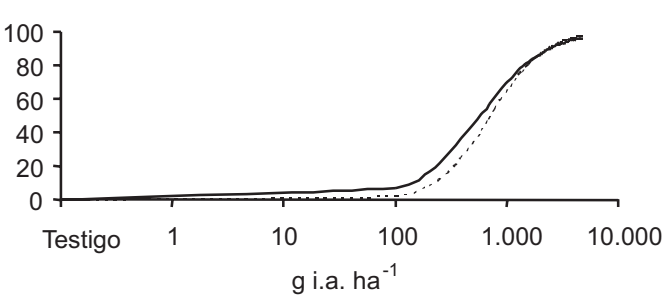

Estado vegetativo: $100+(0-100) /\left(1+(\text { dosis } / 581)^{\wedge} 1,5 \quad r^{2}=0,97\right.$ Estado reproductivo: $100+(-1,1-100) /\left(1+(\text { dosis } / 731)^{\wedge} 1,8 r^{2}=0,95\right.$

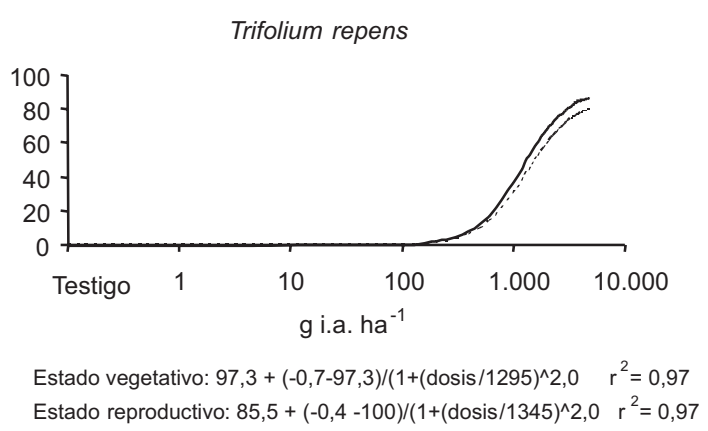

Figura 1 - Curvas dosis-respuesta para las distintas malezas al estado vegetativo y reproductivo. 
interacción significativa entre estados de la maleza. Por este motivo se analizó separadamente cada estado (Tabla1).

A medida que las malezas se incrementan en tamaño se hacen menos susceptibles a los herbicidas (Devlin et al., 1991; Klingaman et al., 1991; Blackshaw \& Harker, 1997). Sin embargo, en este estudio, el estado reproductivo no requirió en todas las especies una mayor dosis para controlar las malezas, probablemente debido a que son malezas tolerantes independientemente de su estado de desarrollo. En el estado vegetativo, la mayor $I_{50}$ correspondió a $I$. diffusa mientras que $O$. indecora y $R$. paraguayensis mostraron la menor $\mathrm{I}_{50} \mathrm{Al}$ estado reproductivo nuevamente I. diffusa presentó mayor $\mathrm{I}_{50}$ y la menor se determinó para $O$. indecora.

Tabla $1-\mathrm{I}_{50}$ de las diferentes especies de malezas al estado vegetativo y reproductivo

\begin{tabular}{|l|c|c|}
\hline \multicolumn{1}{|c|}{ Especie } & $\begin{array}{c}\text { Estado } \\
\text { Vegetativo }\end{array}$ & $\begin{array}{c}\text { Estado } \\
\text { Reproductivo }\end{array}$ \\
\hline Convolvulus arvensis & $968,3 \mathrm{~b}$ & $1571,0 \mathrm{~b}^{*}$ \\
\hline Iresine diffusa & $2078,5 \mathrm{~d}$ & $2322,8 \mathrm{c}$ \\
\hline Oenothera indecora & $547,2 \mathrm{a}$ & $612,3 \mathrm{a}$ \\
\hline Parietaria debilis & $1393,0 \mathrm{c}$ & $1507,0 \mathrm{~b}$ \\
\hline Rumex paraguayensis & $324,7 \mathrm{a}$ & $1320,0 \mathrm{~b} *$ \\
\hline Trifolium repens & $1200,5 \mathrm{bc}$ & $1385,2 \mathrm{~b}$ \\
\hline
\end{tabular}

Para una misma columna, en cada estado de la maleza, letras distintas indican diferencias significativas según una prueba de LSD $(P=0,05)$. Para cada especie, $*$ indica diferencias significativas entre estados

La dosis necesaria para controlar el 90\% con respecto a un testigo sin control de la biomasa de las especies fue mayor para I. diffusa en ambos estados y menor para O. indecora (Tabla 2). Para ambos estados de T. repens no se logró el $90 \%$ en el rango de dosis aplicadas. Esta especie fue pobremente controlada con glifosato a altas dosis en otro trabajo (Puricelli et al., 2004). I. diffusa y $T$. repens son especies perennes y se ha determinado un bajo control de este grupo de especies en otros estudios (Bradley et al., 2004; Whaley \& Vangessel, 2002).

Nuestros resultados indican que el control con la dosis de uso de glifosato puede no ser
Tabla 2 - Dosis necesaria (g.i.a. ha ${ }^{-1}$ ) para obtener el 90\% de control con respecto a un testigo sin control

\begin{tabular}{|l|c|c|}
\hline \multicolumn{1}{|c|}{ Especie } & $\begin{array}{c}\text { Estado } \\
\text { Vegetativo }\end{array}$ & $\begin{array}{c}\text { Estado } \\
\text { Reproductivo }\end{array}$ \\
\hline Convolvulus arvensis & $2210 \mathrm{c}$ & $3400 \mathrm{c}$ \\
\hline Iresine diffusa & $3820 \mathrm{~d}$ & $3930 \mathrm{~d}$ \\
\hline Oenothera indecora & $840 \mathrm{a}$ & $1650 \mathrm{a}$ \\
\hline Parietaria debilis & $2500 \mathrm{c}$ & $2550 \mathrm{~b}$ \\
\hline Rumex paraguayensis & $1100 \mathrm{~b}$ & $3410 \mathrm{c}$ \\
\hline
\end{tabular}

Para una misma columna, letras distintas indican diferencias significativas según una prueba de $\operatorname{LSD}(\mathrm{P}=0,05)$. En ambos estados de desarrollo de Trifolium repens no se logró el $90 \%$ en el rango de dosis aplicadas.

aceptable dependiendo del espectro de malezas y del estado de desarrollo de las mismas, presentes en el barbecho entre cultivos de verano en la región sojera central de Argentina.

\section{LITERATURA CITADA}

BLACKSHAW, E. B.; HARKER, N. Scentless chamomile (Matricaria perforata) growth, development, and seed production. Weed Sci., v. 45, n. 5, p. 701-705, 1997.

BRADLEY, K. W.; HAGOOD, E. S.; DAVIS, P. H. Trumpetcreeper (Campsis radicans). Control in double-crop glyphosate-resistant soybean with glyphosate and conventional herbicide systems. Weed Technol., v. 18, n. 2, p. 298-303, 2004.

CASAFE. Guía de productos fitosanitarios para la República Argentina. 13.ed. Buenos Aires: Cámara de Sanidad Agropecuaria y Fertilizantes, 2007. Tomo 1. p. 1064.

CHAVES, H.; SABATTINI, M. R.; PURICELLI, E. Uso de dosis reducidas de herbicidas para el control de correhuela (Convolvulus arvensis L.). In: REUNIÓN BINACIONAL DE ECOLOGÍA, 1.; REUNIÓN ARGENTINA DE ECOLOGÍA, 20; REUNIÓN DE LA SOCIEDAD DE ECOLOGÍA DE CHILE, 10., Bariloche, 2001. Anais... Bariloche: 2001

DERKSEN, D. A. et al. Impact of post-emergence herbicides on weed community diversity within conservation-tillage systems. Weed Res., v. 5, n. 2, p. 311-320, 1995

DEVLIN, D. L.; LONG, J. H.; MADDUX, L. D. Using reduced rates of postemergence herbicides in soybeans (Glycine max). Weed Technol., v. 5, n. 4, p. 843-840, 1991

FACCINI, D. et al. Control de Plantago tomentosa, Rumex crispus y Urtica urens con distintas dosis de herbicidas preemergentes. Soja Siembra Directa. AAPRESID, n 76, p. 30-32, 2005. 
FACCINI, D.; PURICELLI, E. Eficacia de herbicidas según la dosis y el estado de crecimiento de malezas presentes en un suelo en barbecho. Agriscientia, v. 24, n. 1, p. 29-35, 2007.

KLINGAMAN, T. E.; KING, C. A.; OLIVER, R. L. Effect of application rate, weed species, and weed stage of growth on imazetaphyr activity. Weed Sci., v. 40, n. 2 , p. 227-232, 1991.

PAPA, J. C. Malezas novedosas de importancia emergente con baja susceptibilidad a herbicidas. Soja en Siembra Directa. AAPRESID, v. 14, p. 109-112, 2003.

PURICELLI, E.; TUESCA, D. Riqueza y diversidad de malezas en trigo y barbechos de secuencias de cultivos resistentes a glifosato. Agriscientia, v. 22, n. 1, p. 69-78, 2006.

PURICELLI, E. et al. Control de Trifolium repens con distintas dosis de herbicidas. Soja en Siembra Directa. AAPRESID, n 70, p. 39-40, 2004.

PURICELLI, E. et al. Control de Oenothera indecora y Oenothera affinis con distintas dosis de herbicidas postemergentes. Agromensajes, n. 15, p. 3-4, 2005.

PURICELLI, E.; PAPA, J. C. Parietaria debilis growth in fallow and undisturbed environments. Weed Res., v. 46, n. 1, p. 1-9, 2006.
SCHÜTTE, G; STACHOW, U.; WERNER, A. Agronomic and environmental aspects of the cultivation of transgenic herbicide resistant plants. Berlin: Petra Apel, 2004. 111 p.

SEEFELDT, S. S.; JENSEN, J. E.; FUERST, E. P. Log-logistic analysis of herbicide rate-response relationships. Weed Technol., v. 9, n. 2, p. 218-227, 1995.

STREIBIG, J. C.; RUDEMO, M.; JENSEN, J. E. Doseresponse curves and statistical models. In: STREIBIG, J. C.; KUDSK, P. (Eds.), Herbicide bioassays. Boca Raton: CRC Press, 1993. p. 30-55.

VANLIESHOUT, L. A.; LOUX, M. M. Interactions of glyphosate with residual herbicides in no-till soybean (Glycine max) production. Weed Technol., v. 14, n. 3, p. 480-487, 2000 .

WHALEY, C. M.; VANGESSEL, M. J. Horsenettle (Solanum carolinense) control with a field corn (Zea mays) weed management program. Weed Technol., v. 16, n. 2, p. 293-300, 2002.

WESTWOOD, J. H.; WELLER, S. C. Cellular mechanisms influence differential glyphosate sensitivity in field bindweed (Convolvulus arvensis) biotypes. Weed Sci., v.45, n. 1, p. 2-11, 1997.

ZOSCHKE, A. Toward reduced herbicide rates and adapted weed management. Weed Technol., v. 8, n. 2, p. 376-386, 1994. 